Foundations of physics (New York) 25, 1995, nº1 (january), 183-204.

\title{
The nature of Einstein's objections to the Copenhagen interpretation of quantum mechanics
}

\author{
Michel PATY*
}

SUMMARY.

In what follows, I examine three main points which may help us to understand the deep nature of Einstein's objections to quantum mechanics. After having played a fundamental pioneer role in the birth of quantum physics, Einstein was, as it is well known, far less enthusiastic about its constitution as a quantum mechanics and, since 1927, he constantly argued against the pretention of its founders and proponents to have settled a definitive and complete theory. I emphasize first the importance of the philosophical climate, which was dominated by the Copenhagen orthodoxy and Bohr's idea of complementarity : what Einstein was primarily reluctant to was to accept the fundamental character of quantum mechanics as such, and to modify for it the basic principles of knowledge. I thus stress the main lines of Einstein's own programme in respect to quantum physics, which is to be considered in relation with his other contemporary attempts and achievements. Finally, I show how Einstein's arguments, when dealing with his objections, have been fruitful and some of them still worthy, with regard to recent developments concerning local non-separability as well as concerning the problems of completeness and accomplishment of quantum theory.

\footnotetext{
* Equipe REHSEIS (Recherches épistémologiques et historiques sur les sciences exactes et les institutions scientifiques), CNRS (27 rue Damesme, F-75013 PARIS).
} 


\section{INTRODUCTION}

The true nature of Einstein's well-known opposition to the standard interpretation of quantum mechanics has most often been underestimated or misunderstood. Most of the quantum physicists, i.e. physicists working in the domain of quantum phenomena (condensed matter, atomic, nuclear and particle physics) seem to admit that in the Einstein-Bohr dialogue it was indeed Bohr who was right and that Einstein's position was, as he himself expressed in his own terms, "an effect of fossilisation"1. Max Born's commentaries to the edition of his correspondence with Einstein witness this incomprehension which has lasted since the actual establishment of quantum mechanics, i.e. the years 1925-1927. "The Einstein of to-day is changed", Born wrote in the Schilpp volume, and he opposed Einstein late saying in 1944 that, contrary to Born believing in a God playing dices, he (Einstein) tried "to grasp in a wildly speculative way" the "perfect laws in the world of things existing as real objects"2, to the young Einstein who "used probability as a tool for dealing with nature just like any scientific device"3, and who was an empirist as much as Born and his quantum colleagues were now. Obviously there was here a deep misunderstanding, the nature of which I have discussed elsewhere, about the true nature of Einstein's "constructive" contributions to quantum theory4. Other outstanding physicists who contributed to the edification of quantum mechanics, such as Heisenberg, for example, had similar conceptions (Pauli, although he adhered to the orthodox position, had a more nuanced understanding of Einstein's dissatisfaction with quantum mechanics). At best, they did not understand why one of the most prominent pioneers of quantum physics did not accept the theory when it established itself on what they thought were firm bases and manifested itself as the most powerful theory in the history of physics. Among the physicists of the next generation, who shared this conviction, received from their masters, Pais' account of the subject is typical. His book, Subtle is the Lord, which is probably the most complete and authoritative scientific biography of Einstein, and which deals with great detail with Einstein's contributions to early quantum theory, is rather succinct when he considers the case of Einstein's disease with quantum mechanics, and, in my opinion, diminishes the interest of Einstein's objections as regards improvements in our comprehension of quantum mechanics. Speaking of the Einstein-PodolskyRosen paper of 1935, Pais concludes that it "contains neither a paradox nor any flaw of logic" (I agree with that part of the statement) and that : "it simply concludes that objective reality is incompatible with the assumption that quantum mechanics is complete. This conclusion has not affected subsequent developement in physics, and it is doubtful that it ever will." And Pais adds the following : "He (Bohr) did not believe that the Einstein-Podolsky-Rosen paper called for any change in the interpretation of quantum mechanics. Most physicists (myself included) agree with this opinion." 5

${ }^{1}$ Letter to Max Born, 7.11.1944, in Einstein, Born, 1969.

2 Letter to Max Born, 7.11.1944, in Einstein, Born, 1969.

3 Born (1949), in Schilpp 1949, p. 176.

4 Paty, to be published, chap. 3.

5 Pais 1982, p.456. 
The aim of the present paper is, contrary to these "dominant" conceptions, to propose a different understanding of Einstein's objections to quantum mechanics, in giving Einstein the credit that his position can be different from a mere adherence to a conservative and obsolete view on physics. This aim is twofold. It has first a historical dimension, because we need, in order to grasp the very essence of Einstein's objections, to place them in the context in which they have been formulated, which can be characterized grossly as the "complementarity context"; we need also to regard Einstein's argumentation on quantum mechanics in the light of his own research programme in physics at the same time. The other aim of this paper is more epistemological, or even physical, although it is connected also with historical matters : contrary to Pais' opinion, the objections of Einstein did have an effect on our understanding of quantum mechanics, and I will essentially concentrate on that point. This "positive" effect consists of making us realize the nature of a specific quantum concept which had not been fully perceived as such before Einstein's argumentation and the developments permitted by it : this concept is non-separability, or, more precisely, local non-separability.

\section{THE CONTEXT OF COMPLEMENTARITY}

I will not enter into many details about the context of complementarity, to which Einstein reacted by his objections to quantum mechanics and its interpretation, as I have dealt elsewhere with that topic ${ }^{6}$. I will merely summarize a few elements of the analysis.

The edification of quantum (or wave) mechanics as a formal theory has been very fast, in the years 1924-1927. Its starting point was Einstein's 1917 semiclassical theory, and it kept the two features Einstein was dissatisfied with, as he expressed in that paper: the wave-particle duality of light, and the probabilistic or, equivalently for him, the statistical, character of the predictions. Einstein had hoped that, in this last respect, the theory in progress would provide a causal prediction for the amplitudes of emission and absorption; this was not really the case, at least from a fundamental point of view, as the quantum theory of radiation, which was being developped at the same time, based itself on the quantum mechanical formalism. Now, the theory, presented in a formal way, was considered by its proponents to be definitive and complete, "not subject to any further modification", in the terms of Born and Heisenberg7. It included the two previous "weak points" as cornerstones of the formal building, which Bohr's conception of complementarity, presented at the 1927 Solvay Conference, justified from a fundamental point of view, calling for a new philosophy of knowledge. At this stage, we meet also with the problem of "interpretation", which appears, in the view of the Copenhagen-Göttingen school, as physically necessary to complement an abstract mathematical formalism, but which was, in fact, a mixture of physical intepretation of axiomatic statements and mathematical symbols and of philosophical propositions such as the principle of complementarity itself (and also the consideration that physical reality, in Heisenberg's own words, is only defined "at the very moment of observation"8).

One can then follow the series of objections put forward by Einstein, which

\footnotetext{
6 Paty 1985, et to be published.

7 Born and Heisenberg 1928.

8 Heisenberg, in the discussion, in Electrons et photons, 1927.
} 
seem to evolve according to the periodisation suggested by Pais ${ }^{9}$ : from 1927 to 1931, Einstein tried to show some inconsistency in quantum mechanics, taking uncertainty relations in order to demonstrate, by physical examples, that they cannot be as absolute as claimed by quantum mechanics; the, from 1931 up to his death, Einstein did not look any more for inconsistencies but tried to show that quantum mechanics was incomplete. In fact, throughout all these years, it is the problem of completeness of the theory that Einstein had essentially in his mind, and one can say that, even when he was looking for inconsistencies, it was above all inconsistencies in view of the claim of completeness that he contemplated.

These arguments culminate with the famous EPR paper, supposedly refuted shortly afterwards by Bohr ${ }^{10}$. By completeness, the authors of the paper meant that any element of the physical reality which can be predicted with certainty can be put in correspondence with an element of the physical theory. What they had in mind was the pretention of quantum mechanics to deal thoroughly with the object it aimed at. We shall come back to the EPR reasoning, showing that Einstein and his collaborators, by arising in that manner the question of completeness, did in fact, rightly and explicitly, put their finger on the non-local, or locally nonseparable, character of quantum mechanics. Actually, it is Einstein alone who himself, in his own and later writings, fully explicited this concept. The EPR collaboration, as well as Einstein later on, demanded local separability as a requirement for interaction-independant elements of physical reality, or physical systems, although, as we know now, the recent developments have shown that physical reality is, indeed, not locally separable - and quantum mechanics, in this respect, does not contain the defect of incompleteness. (The question of completeness of quantum mechanics as yet is not resolved, due to the persistence of the problem of quantum measurement ${ }^{11}$.) But the refusal of any property of nonlocality or non separability does not preclude Einstein's priority in having clearly pointed out quantum local non-separability, the importance (and novelty, although it was already contained in the quantum formalism) of which I shall underline further on $^{12}$.

\section{EINSTEIN'S PROGRAMME FOR THE QUANTUM THEORY}

Pais quotes in his book a sentence of Einstein praising in 1926 two Schrödinger's papers on quantum rules, and he adds : "It was the last time he would write approvingly about quantum mechanics."13 Actually this is not true, for Einstein wrote on many occasions positive statements about quantum mechanics. For example, in "Physics and reality" (1936) : "Probably never before has a theory been evolved which has given a key to the interpretation and calculation of such a heterogeneous group of phenomena of experience as has the quantum theory." In many other texts he gave similar appreciations on the heuristic importance of quantum mechanics and its force as a physical theory. In his "Autobiographical notes", written in 1946, published in 1949, he refers to it as "the most successful physical theory of our period, viz., the statistical quantum theory which, about twenty-five years ago, took on a consistent logical form

\footnotetext{
9 Pais 1982.

10 Einstein, Podolsky, Rosen, 1935; Bohr 1935.

11 See, for instance, Paty 1982, 1986, 1988.

12 See also Paty 1986.

13 Pais 1982, p. 442 (Cf. Letter of Einstein to M. Besso, 1.05.1926, in Einstein, Besso, 1972).
} 
(Schrödinger, Heisenberg, Dirac, Born). This is the only theory at present which permits a unitary grasp of experiences concerning the quantum character of micromechanical events". And he adds, significatively (because it sets quantum mechanics on a par with the theory of relativity) : "This theory, on the one hand, and the theory of relativity, on the other, are both considered correct in a certain sense, although their combination has resisted all efforts up to now."14

In 1948, in his article "QuantenMechanik und Wirklichkeit" published in the philosophical journal Dialectica, he states that this theory constitutes an important progress, which can be said in a certain sense definitive, in the knowledge of the physical world15. And in his "Reply to criticisms", in the Schilpp volume (1949) : "I fully recognize the very important progress which the statistical quantum theory has brought to theoretical physics. In the mechanical problems (...), (this theory) even now presents a system which, in its closed character, correctly describes the empirical relations between stable phenomena as they were theoretically to be expected. This theory is until now the only one which unites the corpuscular and ondulatory dual character of matter in a logically satisfactory fashion; and the (testable) relations, which are contained in it, are, within the natural limits fixed by the indeterminacy relations, complete. The formal relations which are given in this theory - i.e., its entire mathematical formalism will probably have to be contained, in the form of logical inferences, in every future theory."16 And again, in 1953 : "I have no doubt that the present quantum theory (or better, "quantum mechanics") is the most perfect theory compatible with experience, in so far as one bases the description on the concepts of material point and potential energy as elementary concepts17".

All these utterances (one could add other ones, taken for example from his correspondence) clearly state that quantum mechanics is a powerful tool, which contains important elements of the ultimate truth : it is thus obviously unfair to say repeatedly that he did not accept the theory. He did not accept it, indeed, as the definitive answer which its proponents claimed it was; that is why it is important to situate his views on quantum mechanics in relation with his own programme of research.

For the positive appreciations just quoted were indeed always mitigated with a restrictive comment, always the same, about the fundamental incompleteness of quantum mechanics, which is most of the times expressed as a feature, not at all of invalidation (quantum conditions must be taken into account in any future theory of atomic processes), but of the impossibility of taking it (its system of concepts and propositions) as a starting point for further developments of a theory which would unify the microcospic and the macroscopic worlds. The quotation we have taken from "Physics and reality" is, for instance, followed by this consideration : "In spite of this, however, I believe that the theory is apt to beguile us into error in our search for a uniform basis for physics, because, in my belief, it is an incomplete representation of real things, although it is the only one which we can build out of the fundamental concepts of force and material points (quantum

\footnotetext{
14 Einstein 1946, p. 81.

15 Einstein 1948.

16 Einstein 1949, p. 666-667.

17 Einstein 1953, p. 6-7.
} 
corrections to classical mechanics). The incompleteness of the representation is the outcome of the statistical nature (incompleteness) of the laws."18 This type of consideration is constant through the texts from the EPR time up to the last writings. Einstein's worries about quantum mechanics do not concern its validation as general law relations with respect to phenomena, but the fundamental problem of principles.

We should have here to recall what were Einstein's conceptions about the fundamental characters of a physical theory (theories of principles, and constructive theories)19. We would then meet with the most important trait of his own programme regarding physical theory in general and of his concern with progresses in the quantum domain in particular. He considered his task to be one of investigation in the fundamental matters, letting aside any other aspects of research not because he considered them as devoid of value. His concern was not to find a theory which "works", but to find a theory deeply rooted in fundamental principles, and this definition of his own research programme does not preclude other types of research work. Already in 1924, in a letter to Maurice Solovine, Einstein said the following about his work : "I had always an interest towards philosophy, but only as a second concern. My interest towards science has in fact always been limited to the study of principles, and this provides the best explanation of all my behaviour. The same reason explains why I published so little, for the strong desire to grasp principles resulted as an effect in fruitless efforts". 20

In his "Preliminary remarks on the fondamental concepts"21 (a rather significant title) in the book of hommage to Louis de Broglie published in 1953, Einstein gives the following precision to show how his own approach is at variance with that of de Broglie : "I nevertheless unceasingly looked for another way of solving the quanta riddle or at least to help preparing the solution. These researches were based on a deep discomfort, of a principle nature ("prinzipieller Natur"), which the bases of the statistical quantum theory inspired me..."

Dealing essentially with principles, Einstein's concern was not to be satisfied with existing theories, i.e. quantum theory and general relativity, but to proceed further towards an integrated theory of principles. Quantum mechanics and relativity theory, although they represent, each for its side, something of the truth about nature, cannot be combined22: here lays, in fact, the essence of the problem, and begins the variety of approaches and beliefs among physicists. Einstein adds to his constatation : "This is probably the reason why among contemporary theoretical physicists there exist entirely differing opinions concerning the question as to how the theoretical foundations of the physics of the future will appear. Will it be a field theory; will it be in essence a statistical theory ?"

Thus, his disatisfaction with quantum mechanics, Einstein expressed it in function of a programme which had to be performed. He was not looking for the

\footnotetext{
18 But this does not mean that incompleteness is to be identified merely with indeterminism (more on this later)

19 See, in particular, Einstein 1936, p. 96-97, and other texts ("The foundation of physical theory", Einstein 1940, for instance).

${ }^{20}$ Letter to Maurice Solovine, 30.10.1924, in Einstein, Solovine 1956; p. 48-49.

21 "Grundbegriff". Einstein 1953, p. 5.

22 Einstein 1946, p. 80-81: see above.
} 
past but to a further stage of our physical knowledge. "What does not satisfy me in that theory" (quantum mechanics), he wrote in his "Reply to criticisms", "is its attitude towards that which appears to me to be the programmative aim of all physics : the complete description of any (individual) real situation (as it supposedly exists irrespective of any act of observation or substantiation)".23

Here comes the question of the nature of Einstein's programme. It is, generally speaking, a realistic and unifying one. "There is something like the 'real state' of a physical system, which exists objectively, independently if any observation or measurement, and which can in principle be described by the means of expression [whatever they be : this is discussed afterwards. My remark, M.P.] of physics."24 This, for the epistemological side. More precisely, Einstein had a physical programme in mind, which he referred to in the following terms : "Continuous functions in the four-dimensional (continuum) as basic concepts of the theory". "Rigid adherence to this programme can rightfully be asserted to me", he adds as an answer to the accusation of Bohr and Pauli of a "rigid adherence to classical theory", and after having noticed that "there is, strictly speaking, today no such a thing as a classical-field theory" (i.e., a field theory which would be "complete" in a specific sense, i.e. a theory of the field-creating mass, which was, for the physics of fields, Einstein's problem25).

Einstein's programme for quantum physics was thus strongly linked with his programme for the field : for him, quantum as well as field physics were unachieved, and his problem was to achieve them, or better, to accomplish them together. Such an aspect of "completeness", which appears as completeness endowed with a different meaning from the one which is strictly involved in the EPR argument, is nevertheless to be kept in mind when we deal with the restricted sense it has in the EPR paper and, more widely, in consideration of the problem of completeness of quantum mechanics : but it is not to be superimposed to it (contrary to what Popper states in the 3rd volume of his Postcript, on The quantum schism26). There are early indications, from Einstein himself, that he was partly motivated in his unitary field research programme by the preoccupation of resolving the quantum riddle27 (see, in this respect, its "Autobiographical notes").

Since 1920, Einstein was looking for an overdetermined field theory, i.e. a theory in which the field variables would be overdeterminated by appropriate equations whose number would be larger than the number of field variables 28 . He proposed the following reasoning : take general covariance; require agreement of the equations with Maxwell's and gravitation theories; require that the equations which overdetermine the fields have symmetrical static solutions which describe the proton and electron : it then should be so that one obtains conditions of overdetermination which restrict the choice of the initial conditions of the fields. Instead of an under-causality, one would obtain in such a way an overcausality

\footnotetext{
23 Einstein 1949, p.667.

24 Einstein 1953, p.7.

25 Einstein 1949, p. 675.

26 Popper 19??.

27 Einstein 1923. Cf. Pais 1982, p. 464, Stachel, 1986.

28 Letter to M. Besso, 5.1.1924, in Einstein, Besso, 19??. John Stachel has given a profound analysis of this Einstein's expectation (Stachel, 1986).
} 
("Überkausalität")29. Such a programme was praised up to 1925 by Born and others.

To recall here Einstein's views about the relations between quantum mechanics and the theory of relativity exceeds the limits of this article. Let us merely mention that he considered that they were foreign to each other and were not to be fused together, due to the strong difference of their epistemological features. He had many worries about the problems related with quantum field theory (he saw in it a kind of a monster, importing (special-)relativistic features into quantum mechanics, and exhibiting infinities). What interested him, however, in this respect, was not the special but the general relativity theory, as the problem he eventually considered was that of a unification of the dynamics of the atomic matter with the dynamics of gravitation.

What is certain is that, even when dealing with the apparently quite different problem of the continuous field theory, Einstein had in mind the problem of quantum physics, and this was one of his motivations for pursuing his research 30 . Up to the end of his life, his programme was "to find the equations of the total field". Interestingly enough, he never mixed, however, in his scientific contributions, considerations on quantum theory and on the continuous field. This is an attitude he had since his first articles on both qestions, in 1905, and it appears a characteristic feature of his scientific method or style in physical research 31 .

It is only recently that such a programme, namely looking for a total field of matter, has become the main concern of quantum physicists; indeed they are looking for a quantum field, but they encounter, although on the other side of the hill (quantum field, not continuous one), the problem with which Einstein had an endless struggle : the unification of the quantum domain and of general relativity, or gravitation.

\section{THE ROLE OF EINSTEIN'S OBJECTIONS \\ IN THE CLARIFICATION OF QUANTUM CONCEPTS}

Einstein's argumentation concerning the deficiencies of quantum mechanics, throughout the years, is centered around two problems : the probabilistic-statistical character of quantum theory and its inherent non-locality. Such were, in his view, the essential features of its incompleteness. This view was attained by Einstein very soon, although his formulation of the main arguments has somewhat evolved with the time : I would like to show, in particular, in what follows, what has been the evolution of Einstein's argumentation, which has sometimes been, in my opinion, misinterpreted.

I said that Einstein attained very soon the essential features of his criticism against quantum mechanics or, better, against the interpretation of quantum mechanics which considered it as a fundamental and complete theory. It is obvious for probabilities, or "statistical character" - a feature of quantum theory which he noted in 1917 and which further elaborations by the quantum physicists did not modify, although he himself had seen in it a weakness, contrary to a fundamental achievement. Quantum mechanics did only change the status of

29 Einstein 1936, 1940. He stated: "Field theory programme, but not yet performed".

30 Pais rightly notices in his book (Pais 1982, p. 465-466), founding himself in Einstein's correspondance, that the problems of the unified field theory and of quantum physics were simultaneously present to his mind.

31 On this question, see Paty, 1993, and to be published. 
probability, raising it from a mere tool to a principle, through the interpretation of the state function. When he introduced probability in quantum physics, first through a reinterpretation of Boltzmann entropy formula, then through his fluctuation calculations which led him from his 1905 paper on radiation energy quantization to his 1916 synthesis, with radiation momentum, and to his 1925 calculation of monoatomic gas ("Bose-Einstein) statistics, Einstein considered it through its frequency interpretation, as a useful (and powerful) tool to point out some characteristic features of quantum phenomena, that could be attained in that indirect way. He always had the idea that a fundamental quantum theory should overcome this probabilistic character which was, in his view, so to speak, only provisional.

But his demand for determinism, or, better, for a non-statistical character of the theory, is more subtle than what is generally thought, as one can see by looking at his argument of incompleteness. For he did not reject a priori the probabilistic character of the description of physical states as fundamental. He wanted to show (such is the argument in essence) that this description cannot in any case be referred to individual systems (to demonstrate it he used the assertion of a local separability principle). The non-local character of the $\psi$-function, which was a consequence of the non-separability of the correlated sub-systems in a EPR-type situation, which itself resulted from the non-factorizability of the $\psi$-functions in the mathematical formalism of quantum mechanics, entailed the statistical character of the description : for only in this way non-locality for individual systems could be avoided, according to him. As a matter of fact he demonstrated, with his EPR argument, that the probabilistic wave-function could only led to a statistical description, leaving open the problem of the representation of an individual system: in his demonstration, he made use of a separability or locality principle for individual systems which, he recognized, was not present in quantum mechanics.

Thus non-locality plays a role in Einstein's rejection of the fundamental character of quantum mechanical probabilistic assertions, which he expressed in the famous sentence "God does not play dice". Let us now come to this concept, which has required som delay before being completely evidenced and understood.

It is only recently, indeed, that the concept of non-locality as a fundamental feature of quantum mechanics has been fully appreciated, and commentators have seldom realized that this was one of Einstein's main points. Even Bohr did not understand that non-locality was at the root of Einstein's argumentation, and did not properly realize that is was also an inherent feature of quantum mechanics. It is usually thought that it was Bohr who pointed out nonseparability - or, as we now understand it, local non-separability - in his answer to the EPR objections. But in fact it was not so, strictly speaking, and it is in the Einstein-Podolsky-Rosen's paper itself that non-locality is described and that emphasis is put on it. I shall try to clarify that point in what follows. I said that very early Einstein paid attention to it : we can trace it back up to a remark he made about indistinguishibility of quantum particles and the exclusion principle. Answering a criticism made by Ehrenfest and others to his and Bose's papers of 1924-1925, he emphasized the fact that particles are not dealt with, in these papers, as mutually independent, and that their treatment, i.e. the Bose-Einstein counting of particles as indistinguishable,"express(es) indirectly a certain hypothesis on a mutual influence of the molecules which for the time being is of a quite mysterious nature"32.

But non-locality as such is pointed at when macrocospic distances are involved. Hence it would manifest itself in EPR-type situations (in the formalism or in phenomena, would we say today; as for Einstein, as we shall see, it was only in the formalism - if one would extend its interpretation to the description of individual

32 Einstein 1925, p.3 (as quoted in Pais 1982, p. 428). 
systems). Before the EPR paper, however, and as early as the Solvay Conference of 1927, something which looks like what will later be called non-locality is present in Einstein's objections. On that occasion, in the general discussion opened by Bohr who presented his idea (soon to become a "principle", of a philosophical nature, indeed) of complementarity, Einstein expressed his dissatisfaction with the fundamental character of quantum mechanics, by taking the example of electrons being diffracted by a slit and hitting on a screen covered by a photographic plate. He argued that a purely statistical theory was incomplete, because each electron has a trajectory which could be detected at least approximately, and that if one admitted such an individual description of each electron, then one had to face a problem : if $|\psi|^{2}$ is related to an individual particle, expressing "the probability that this particle is located at a given place", that interpretation of the function "implies a quite peculiar mechanism of action at-a-distance, which forbids the wave, continuously distributed in space, to produce an action in two places of the screen". This last sentence refers to a non-locality which seemed to Einstein not acceptable, because it would involve an action-at-a-distance, contrary to special relativity 33 . In this first thought about non-locality, which comes from the wave character of the physical object under consideration, Einstein saw it as contradictory to the particle concept which quantum mechanics utilizes together with the wave concept.

Once again (such contradictory character he pointed out as early as 1909) stating that it would demand an approach towards quantum phenomena which would break with classical theories, Einstein found himself with a feature which was not to be solved simply by asserting that we can deal, from a fundamental point of view, only with the wave and/or with the particle concepts : there is still something more fundamental to find. We deal provisionally with those classical concepts at the cost of using a theory of a purely statistical nature. With the statistical interpretation of the $\psi$-function one is free from any action-at-a-distance belonging inherently to particle non-locality. Such was Einstein's view in 1927, and the essence of his further reasoning is present in this simple argument, which was already aimed at refuting the pretention of quantum mechanics to be a fundamental complete theory. He would even try to do more for some time, and show that quantum mechanics has logical inconsistencies in giving absolute credit to Heisenberg relations. Such is his radiation-in-a-box experiment of 1930, reported by Bohr ${ }^{34}$, which refuted it by invoking General relativity; let us mention en passant that, although Bohr's answer has unanimously been considered to be the right one, it has recently been shown inexact, the argument to keep the fourth indeterminacy relation owing nothing to general relativity, and being of a much more fundamental character ${ }^{35}$. Such is also his article with Tolman and Podolsky of 1931 about "Knowledge of past and future in quantum mechanics"36, where he presents for the first time, without insisting, the idea of correlation of distant particles.

Later on, he would consider that quantum mechanics is free of inner contradictions but is incomplete. It is in trying to show this incompleteness by physical arguments that he made explicit the non-local character of physical systems as described by quantum mechanics We notice, in such attempts, that incompleteness, in Einsteins's view, is not merely to be identified $a$ priori with indeterminism and the statistical character of the description. We shall emphasize further that Einstein's motivation was not primarily to restore determinism against

33 Einstein's intervention in Electrons et photons 1928, p. 255-256.

34 Bohr 1949.

35 See Treder etc.

36 Einstein, Tolman and Podolsky, 1931. 
probabilistic description, but to point out non-locality as a defect of the formalism which lets us with only a statistical description.

Before the EPR paper, Einstein put forward an argument (unpublished and presented only privately) already in 1933, reported later by Léon Rosenfeld. This early consideration, interestingly enough, shows itself as a close prefiguration of the EPR argument ${ }^{37}$.

Rosenfeld tells that, at that time, he thought Einstein meant merely to illustrate by this argument unfamiliar features of quantum phenomena. In fact, emphasis is given in it to the non-local character of quantum phenomena (probability being only present in the background if we keep in mind the 1927 Solvay argument).

The next objection is the EPR reasoning of 1935. The example of two quantum particles having interacted in the past is fully developped in the article as regards its quantum mechanical description. The case is well- known and I will not insist on it, restricting myself to show that, definitely, emphasis is given to the non-local character of the quantum mechanical description, if we consider it as applying to individual events.

To help understand more clearely what is at stake here, I would like to remind that the EPR argument is based on the idea of particle correlation and on the fact that the nature of these correlations is quite different in the classical and in the quantum cases.

\section{CLASSICAL AND QUANTUM CORRELATIONS BETWEEN DISTANT SUBSYSTEMS}

The difference is shown, in the EPR paper ${ }^{38}$, through the question of the determination of conjugate physical quantities such as coordinates and momenta (it becomes eventually immediately clearer if we consider the EPR argument reformulated by David Bohm in his book of 1951, Quantum Theory ${ }^{39}$, by taking as conjugate or incompatible quantities two components of the angular momentum : spin conservation holds as in the classical case $\left(J_{x}(1)=-J_{x}(2), J_{y}(1)=-J_{y}(2)\right.$, $\left.J_{z}(1)=-J_{z}(2)\right)$, but the correlation takes a different form in that it is not possible to have a simultaneous determination of the various components of a unique spin.

At this stage, the specificity of quantum correlations comes from (and shows itself in) the probabilistic definition of the wave function, the noncommutation of the operators which represent the physical quantities considered, the choice made by the act of measurement of one spin component (if we consider this case), at the exclusion of the other ones, i.e., the reduction of the wave packet or reduction process. In summary, it is the fundamental statements (or axioms) of quantum mechanics that make the difference, and their argument took in effect as their target these statements or axioms, when they confronted their consequenceswith the requirements of "local realism", which in fact would correspond to classical type correlations. (Let us notice, however, that it appeared explicitly as "local realism" only afterwards. This local character was implicit in the requirements for "elements of the physical reality".) For the EPR authors, the impossibility for quantum theory to predict correlated physical quantities was an evidence for the incompleteness of that theory. Such was the function of the thought experiment which they invoked : to investigate the deepness and the range of the fundamental statements of quantum theory. But this inquiry was done from outside, as the criterion considered (local realism) was foreign to these statements and could not be determinant if one considered only these statements as pertinent.

\footnotetext{
37 Rosenfeld 1967. Cf. Paty 1985.

38 Einstein, Podolsky, Rosen 1935.

39 Bohm 1951.
} 
Bohr did not express it clearly, but Einstein himself did, for instance in his 1948 article of Dialectica; emphasize that his "separability" principle is not comprehended in the formalism of quantum theory, stating that such a requirement is nevertheless reasonable when one deals with individual physical systems considered in space.

Bohr's answer to the EPR argument did not speak explicitly of nonseparability as it has been understood afterwards, and it did not go further than the EPR description of the phenomenon which exhibited non-separability as a nonlocality. Bohr's answer was so much impregnated with his philosophical preconception of complementarity and his philosophy of observation, that he did not actually state explicitly the quantum concept here at stake, non-separability. It is again Einstein who "translated" in such terms Bohr's answer to the EPR argument. He did it in a very explicit way sometime later, when replying to Bohr's criticism in the Schilpp's volume Albert Einstein philosopher-scientist ${ }^{40}$. "Of the 'orthodox' quantum theoreticians whose position I know", did he write, "Niels Bohr's seems to me to come nearest to doing justice to the problem. Translated into my own way of putting it (my emphasis, M.P.), he argues as follows : "If the partial systems $A$ and $B$ form a total system which is described by its $\psi$-function $\psi(\mathrm{AB})$, there is no reason why any mutually independant existence (state of reality) should be ascribed to the partial systems $A$ and $B$ viewed separately, not even if the partial systems are spatially separated from each other at the particular time under consideration (Einstein's emphasis). The assertion that, in this latter case, the real situation of $B$ could not be (directly) influenced by any measurement taken on $A$ is, therefore, within the framework of quantum theory, unfounded and (as the paradox shows) unacceptable." (By this last sentence, Einstein makes explicit that non-separability is a non-locality).

Returning to the original Einstein-Bohr dialogue (or controversy) of 1935, we can say that what Bohr added to Einstein's previous description in the EPR paper, is merely that there is no reason for any mutual independent existence of substates. But is is only much later on that the acceptation as a fact of these specific quantum correlations justified by the formalism has led to the full acceptation of the specific quantum concept of non-separability (local nonseparability). Nevertheless, the presence of non-separability in the quantum formalism, before any experiment showing it directly, explains, in my opinion, why many physicists have - wrongly - underestimated the importance of Bell's theorem. And the fact that separability is alien to quantum concepts as a whole explains - but, this time, with good reasons - why most of the physicists did believe that quantum correlation would win agains locality before the realization of convincing experiments : they favoured the overall evidence given to quantum theory by so many phenomena. Strictly speaking, however, one could admit that the domain of most of these phenomena is just dumb about locality.

It is indeed this non-separability that expresses the quantum character of the correlation under consideration. Compared with it, the classical correlation is rather light or weak : despite the relation between physical quantities due to the conservation law, the two subsystems of a classical system are totally separable one from the other: a modification of one of them has no effect on the other as soon as they are sufficiently far from each other (local separation) to be free of any mutual interaction. The description of the quantum correlation is not so simple and cannot be done by using images of that kind (billiard balls ... ), because of the difficulty we meet when we want to describe with simple words the definition of physical quantities in terms of spectra and frequencies. It is only when we think quantum correlations in imaged or intuitive terms of the above classical type, that we eventually consider - as many people do - the quantum situation as being the exact

40 Einstein 1949, p. 681-682. 
opposite of the classical situation : in such a view it would express the (instantaneous) modification of one of the subsystems as the effect of a modification of the other despite of their remoteness. One could say, in a way, that Einstein made such a translation ${ }^{41}$. That is why he sent back quantum mechanics to purely statistical interpretation. But such a statement is only a translation, as there is nothing physical that interacts, considering the quantum quantities involved.

Strictly speaking, the quantum correlation is nothing but what is expressed by the formalism of the quantum theory : it is described by quantum mechanics and is entailed by its fundamental statements. It is, precisely, the nonseparability of the state vector which describes the overall system with respect to the state vectors which represents the subsystems : from the point of view of the formalism, this property of the state vectors is their non-factorizability. Unless we modify the initial conditions (which is not possible in a causal way), we cannot separate (split) the wave fonctions of the subsystems into independent eigenfunctions - if we restrict ourselves to the description of systems by quantum mechanics. When one of the subsystems (electron, photon ... ) is submitted to a measurement which determines its spin state, this corresponds to a change of initial condition (and subsequently to a change of the problem) : the initial condition, is henceforth the electron (photon ... ) singled out, with the exactly determined value of its measured spin.

It is useful to clarify here a trait of Einstein's reasoning with the EPRtype thought experiment which has largely been misunderstood. It has to deal with a difference in the expression of the argument between the "three-men" EPR redaction and Einstein's own way of putting it. Einstein expressed in a letter to Schrödinger his dissatisfaction with the writing-up of the EPR paper - donr in fact done by Podolsly, because of his better familiarity with English -, which he thought clumsy in some respect, saying that "the main point has been buried under erudition"42. Although some commentators have taken profit from this avowal that EPR argument was not of Einstein, it seems clear that its essence is of his own, from the idea of quantum particles correlation to avoid the perturbation argument generally invoked to justify uncertainty relations, up to the conclusion about the incompleteness of the quantum description ${ }^{43}$. But there is a difference between the emphasis given in the two expositions to the fundamental reason for incompleteness.

The EPR paper insists on the determination of the quantum variables, such as coordinate and momentum, which ultimately provide the state vector. Without measuring them, one can afford them "reality", by making use of the measurement on the other together with the relation of conservation entailed from the initial state. Thus, EPR stated, quantum mechanics says nothing of such elements of reality, and is therefore incomplete - from which many people have thought, afterwards, that hidden variables were implicit in such a resoning to recover those lost elements of reality 44 .

When Einstein explained the argument in his own terms, he emphasized not what happened to the variables but what was the description of the state supposed to represent the real system. This consideration was present also in the EPR paper, but did not seem - buried under erudition - so central as it is in Einstein' true thought. A complete measurement of a complete commuting set A of quantities of the first subsystem, he stated, defines not only the $\psi$-function of it $\left(\psi_{I}^{A}\right)$, but

41 Cf. Einstein 1949, p. 682.

42 Einstein, letter to E. Schrödinger, aug. 1935 (Einstein's Archives).

43 cf. Howard 1986.

44 See Paty 1993. 
also, through the correlation coming from the initial state, the $\psi$-function of the second $\left({ }_{\Psi I}^{A}\right)$, without any measurement having been performed on this last one. But the same can be said with a set $B$, different from and incompatible (in the sense of q.m., that is through anticommution relations and thereof Heisenberg inequalities) with $A$, leading to another determination, without direct measurement, of the state of the second system $\left({ }_{\psi_{I I}^{B}}^{B}\right)$. As these two states have no reason to be the same, there is no unique, non-ambiguous correspondence between the state of a system and its theoretical description, unless one admits an (inacceptable, for Einstein) instantaneous influence at-a-distance of what happenned to the first system on to the second, i.e. Einstein's transcription of non-locality. As this reasonoing was done for the consideration of individual systems, one must admit, according to Einstein, that the $\psi$-function does not describe an individual system: it is only related to ensembles of systems. It is for that reason that, to him, quantum mechanics is incomplete. Separability and locality (independence of systems differently located in space) are essential in such a statement. That is to say, apparent non-separability or non-locality is, for him, the reason of incompleteness: not indeterminism, which is merely a consequence ${ }^{45}$.

\section{NON-LOCALITY AS A CRITERION AND AS A PHYSICAL FACT}

The quantum definition of non-separability, already present in the formalism of quantum mechanics, became explicit as an effect of the discussion between Einstein, Podolsky, Rosen, and Bohr. But it remained optional : it was possible to imagine in principle a modification of the theory which would eliminate it. As such, it was, for those who were not content with this theory (because, in the case of Einstein, of its non-local property), a manifestation of its incompleteness (because it obliged to refer the $\psi$-function not to a single event but only to a statistical ensemble). But this consideration on non-separability or non-locality, if one accepted, as the standard quantum mechanics did, that trough $\psi$-function the theory described individual systems as well as ensembles, was, in the context of the time, hidden under the apparently more general problem of causal determination, also in debate.

The possibility to restore determinism had been considered far before the concept of non-separability came in the forefront. And it still was the aim of all attempts at alternative descriptions of microsystems even after the EPR argument, until recently; indeed the EPR "paradox" strengthened the motivation for those attempts but the very essence of the problem remained unperceived, up to the outstanding work of J.S. Bell in 1964.

At this point, we should evoke the various attempts at showing an incompatibility between quantum mechanics and deterministic hidden variables which had been tried, since Von Neumann. I will skip all that part of the story, and arrive to Bell's work around 1964.

When looking for the proof of incompatibility between such theories and quantum mechanics, Bell realized that there was an entire class of hidden variables which was incompatible with quantum mechanics : the local class. He had been put on the way by the consideration by David Bohm in 1952 of a possible model which would be causally deterministic and non-local, and had been able to show that all the previously proposed proofs of incompatibility were unconclusive, being based on restrictive hypothese (in his 1966 paper, written before the 1964

45 For all this description and analysis of Einstein's argumentation, see Paty (to be published). 
one $\left.^{46}\right)$. His own criterion of locality, which he intended to demarcate quantum mechanics from deterministic supplementary parameters ${ }^{47}$, was discovered by him while he meditated on the EPR thought-experiment as reformulated by Bohm and by Bohm and Aharonov, and particularly on the following sentence by Einstein, commentating in his "Autobiographical notes" the EPR situation : "But on one supposition we should, in my opinion, absolutely hold fast : the real factual situation of the system $S_{2}$ is independent of what is done with the system $S_{1}$, which is spatially separated from the former." 48 We have to notice also that the measurement problem is indeed present in these formulations of locality.

When we consider retrospectively the very content of Einstein's argumentation, we cannot omit taking into account that, nowadays (and very recently), non-locality in quantum physics has been given a fundamental, and well probably definitive, status : it has been raised to the state of a physical fact ${ }^{49}$, and it is founded on a conceptual ground which is more general than the question of determinism and hidden supplementary variables. And the origin of the concept is to be found nowhere else than in Einstein's argumentation when he objected against quantum mechanics. True, Einstein disliked such a concept and saw in it an evidence for the incomplete character of the theory ${ }^{50}$. But this does not preclude in any way that he has indeed pointed out the concept.

To conclude, let us state here that non-locality is part if the quantum theory. There remains, however, a question which is by now unsolved, and it is that of the completeness of quantum mechanics. Insofar as local non-separability is a physical fact, it cannot be seen any more as a weak point in a theory; but there seems to remain, indeed, still a weak point in quantum mechanics, which is the problem of measurement (and this problem shows itself in our formulation of nonlocal phenomena). We thus can dissociate Einstein's considerations on non-locality and on the completeness of the theory : the first one has been useful as we have seen, for the explicitation of this quantum concept ${ }^{51}$, and the last one is still of

46 Bell 1966. On these circumstances, see Jammer 1974.

47 Bell 1964.

48 Einstein 1949, p. 84-85.

49 See Paty 1982, 1986 and 1988, for argumentation and bibliography.

50 See for exemple the continuation of Einstein's remark on non-locality which we have quoted above ("Autobiographical notes", Einstein 1949, p. 682) : "By this way of looking at the matter it becomes evident that the paradox forces us to relinquish one of the following two assertions : 1) the description by means of the $\psi$-function is complete; 2) the real states of spatially separated objects are independant of each other."

51 It has been argued by Don Howard (1986) that Einstein's position has evolved towards an acceptation on "non-separability" although he always maintained his opposition to "non-locality". I agree on one point, which is that the use of each of the two terms can be characterized differently (I myself stated it in Paty 1982), but non-separability and non-locality are one and the same concept of quantum physics, which can be called unambiguously "local non-separability". Although it should be argued in more details, which I have no place to do here, I maintain that a sharp distinction like Howard's one cannot be found in Einstein's writings, even in Einstein 1948 which gives the clearest definition of his understanding of these concepts. Clearly - in my opinion -, Einstein considers that non-separability and non-locality are one and the same concept, because quantum mechanics deals, in its statements, when they refer to physical situations, also with the problem of measurement. Non-locality, as it stands in the definition used by Howard, corresponds to the criterion of non-locality proposed by Bell following Einstein's considerations. It seems to me that the concept in itself is more general than the criterion, which refers specifically to observational situations, and that non-locality is a quantum-mechanical concept which is now raised to the status of a physical fact (see Paty 1986), without any reference to a transmission of a physical influence between two spatially separated subsystems. On the contrary, Howard definition seems to equate non-locality to such an action-at-a-distance, which leads to an oversimplification 
actuality.

\section{REFERENCES}

Bell, John S. (1964). On the Einstein-Podolsky-Rosen paradox, Physics 1, 1964, 195-200. Repris in Bell 1987, pp. 14-21.

- (1966). On the problem of hidden variables in quantum mechanics, Review of Modern Physics 38, 1966, pp. 447-452. Repris in Bell 1987, pp. 1-13.

- (1987). Speakable and unspeakable in quantum mechanics, Cambridge University Press, Cambridge, 1987.

Bohm, David (1951). Quantum theory, Prentice Hall, Englewood Cliffs, 1951.

Bohr, Niels (1935). Can quantum mechanical description of physical reality be considered complete ?, Physical Review 48, 1935, 696-702.

- (1949). Discussion with Einstein on epistemological problems in atomic physics, in Schilpp 1949, pp. 199-242.

von Borzeskowski, Horst-Heino and Treder, Hans-Jürgen : The meaning of quantum gravity, Reidel, Dordrecht, 1988.

Born, Max and Heisenberg, Werner (1928). La mécanique des quantas, in Electrons et photons 1928, pp. 143-183.

Born, Max (1949). Einstein's statistical theories, in Schilpp 1949, pp. 161-178.

Einstein, Albert (1923). Bietet die Feldtheorie Möglichkeiten für die Lösung des Quantumproblems ?, Preussische Akad. Wissenschaften, Phys. Math. Klasse, Sitzungsberichte, 1923, pp. 359-364.

- (1925). Quantentheorie des einatomigen idealen Gases. Zweite Abhandlung, Preussische Akad. Wissenschaften, Phys. Math. Klasse, Sitzungsberichte, 1925, 3-14.

-, Tolman, R.C. and Podolsky, Boris (1931). Knowledge of past and future in quantum mechanics, Physical Review 37, 1931, 780-781.

-, Podolsky, Boris and Rosen, Nathan (1935). Can quantum mechanical description of physical reality be considered complete ?, Physical Review 47, 1935, 777-780.

- (1936). Physik und Realität, engl. transl.: Physics and reality, in Einstein 1954 (ed. 1981, pp. 283-314).

- (1940). The fundaments of theoretical physics, in Einstein 1954 (ed. 1981, pp. 315-126).

- (1946). Autobiographishes. Autobiographical notes, in Schilpp 1949, pp. 1-94.

- (1948). Quantenmechanik und Wirklichkeit, Dialectica 2, 1948, 320-324.

- (1949). Reply to criticism. Remarks concerning the essays brought together in this co-operative volume, in Schilpp 1949, pp. 665-688.

- (1953). Einleitende Bemerkungen über Grundbegriffe. Remarques préliminaires sur les principes fondamentaux, in Louis de Broglie, physicien et penseur, Albin Michel, Paris, 1953, pp. 4-15.

- (1954). Ideas and opinions, new transl. and revision by Sonja Bargmann, Crown, New York, 1954 ( Repr. Laurel, New York, 1981). 
- (1956). Briefe an Maurice Solovine. Lettres à Maurice Solovine, Gauthier-Villars, Paris, 1956.

- et Besso, Michele (1972). Correspondance, 1903-1955, publiée par P. Speziali (original texts and french transl.), Hermann, Paris, 1972.

-, Born, Max (1969). Briefwechsel 1916-1955, Nymphenburger Verlagshandlung, Munich, 1969. Engl. transl. The Born-Einstein letters, ed. by M. Born, Walker, New York, 1971.

Electrons et photons. Rapports et discussions du cinquième Conseil de physique tenu à Bruxelles du 24 au 29 octobre 1927, sous les auspices de l'Institut International de Physique Solvay, Gauthier-Villars, Paris, 1928.

Howard, Don (1986). Einstein on locality and separability, Studies in history and philosophy of science 16, 1985, 171-201.

Jammer, Max (1974). The philosophy of quantum mechanics. The interpretations of quantum mechanics in historical perspective, Wiley and sons, New York, 1974.

Pais, Abraham (1982). Subtle is the Lord. The science and life of Albert Einstein, Oxford University Press, Oxford, 1982.

Paty, Michel (1982). L'inséparabilité quantique en perspective, Fundamenta Scientiae 3, 1982, pp. 79-92.

- (1986). La non-séparabilité locale et l'objet de la théorie physique, Fundamenta Scientiae 7, 1986,47-87.

- (1985). Einstein et la complémentarité au sens de Bohr. Du retrait dans le tumulte aux arguments d'incomplétude, Revue d'histoire des sciences 38, 1985, 325-351.

- (1988). La matière dérobée, Archives contemporaines, Paris, 1988.

- (1993). Sur les variables cachées de la mécanique quantique: Albert Einstein, David Bohm et Louis de Broglie, La Pensée, n 292, mars-avril 1993, 93-116.

- (to be published). Einstein, les quanta et le réel.

Popper, Karl (1983). The quantum schism, volume 3 of The Postcript to the Logic of Scientific Discovery, Hutchinson, London, 1983.

Rosenfeld, Léon (1967). Niels Bohr in the thirties : consolidation and extension of the conception of complementarity, in S. Rosental(ed.), Niels Bohr, his life and work, Wiley and sons, New York, 1967.

Schilpp, Paul-Arthur (ed.) (1949). Albert Einstein, philosopher-scientist, The Library of living philosophers, Evanston (Ill.), 1949.

Stachel, John (1986). Einstein and the quantum : fifty years of struggle in R. Colodny (ed.), From quarks to quasars, University of Pittsburgh Press, Pittsburgh, 1986. 